\title{
Manuelle Therapie nach Mulligan zur Verbesserung der Knieflexion bei Patienten nach Knietotalendoprothese
}

\author{
Randomisierte kontrollierte Studie
}

\section{Manual Therapy according to Mulligan to Improve Knee Flexion in Patients after Total Knee Arthroplasty}

\author{
Randomised Controlled Trial
}

\author{
Autoren \\ Deniz Halil \\ Institut \\ maxQ. - im bfw - Schule für Physiotherapie Iserlohn \\ Schlüsselwörter \\ Knietotalendoprothese, Knie-TEP, Manuelle Therapie, \\ Mobilisation with Movement, MWM, Mulligan, ambulante \\ Rehabilitation \\ Keywords \\ total knee arthroplasty, knee replacement surgery, manual \\ therapy, mobilisation with movement, MWM, Mulligan, \\ outpatient rehabilitation \\ eingereicht 20.6.2017 \\ akzeptiert $\quad 2.8 .2017$ \\ Bibliografie \\ DOI https://doi.org/10.1055/s-0043-124536 \\ manuelletherapie 2018; 22: 34-43 \\ (c) Georg Thieme Verlag KG Stuttgart · New York \\ ISSN 1433-2671 \\ Korrespondenzadresse \\ Deniz Halil \\ Oesestr. 33 \\ 58675 Hemer \\ E-Mail: dhali@@web.de
}

\section{ZUSAMMENFASSUNG}

Patienten nach Implantation einer Knietotalendoprothese (TEP) benötigen zur Bewältigung ihres Alltags zügig eine ausreichende Gelenkbeweglichkeit. Evidenz für manualtherapeutische Techniken zur Förderung der Beweglichkeit in der Rehabilitationsphase nach Knie-TEP wurde bisher nicht veröffentlicht. Das primäre Ziel dieser monozentrischen randomisierten kontrollierten Pilotstudie war zu untersuchen, wie sich die Anwendung der manualtherapeutischen Technik „Mobilisation with Movement“ (MWM) nach dem Mulligan-Konzept [1] [2] auf die Funktion der Knieflexion nach primärer Knie-TEP auswirkt. Primärer Zielparameter war die Mittelwertdifferenz der aktiven Flexion, sekundärer Zielparameter die Mittelwertdifferenz der aktiven Extension, Schmerzreduktion und Funktionsstatus. Beim primären Zielparameter bestand ein statistisch signifikanter Unterschied zwischen der Interventions- und der Kontrollgruppe. Der sekundären Zielparameter Schmerzlinderung zeigte ebenfalls einen signifikanten Unterschied zugunsten der Interventionsgruppe. Bei den weiteren sekundären Zielparametern fanden sich keine signifikanten Unterschiede.

Ein statistisch und klinisch signifikanter Effekt zugunsten der MWM-Technik gegenüber rotatorischen Mobilisationstechniken konnte nachgewiesen werden. Zur Bestätigung der Effekte sind weitere Studien mit höheren Fallzahlen und Follow-ups nötig.

\section{ABSTRACT}

After implantation of total knee arthroplasty patients need to quickly develop adequate joint mobility in order to be able to cope with their daily activities. To date no evidence for the use of manual therapy techniques to support joint mobility in the rehabilitation phase after total knee arthroplasty has been published. The primary objective of this mono-centred randomised controlled trial was to examine the effect of the application of the manual therapeutic technique "mobilisation with movement" (MWM) according to the Mulligan Concept [1] [2] on knee flexion following primary total knee arthroplasty. Primary outcome measure was the mean value difference of active flexion. The secondary outcomes were the mean value difference of active extension, pain reduction and functional status.

Regarding the primary outcome measure there was a statistically significant difference between the intervention and the control group. The secondary outcome pain reduction also showed a significant difference in favour of the intervention group. Further secondary outcome measures showed no significant differences. 
A statistically and clinically significant effect in favour of MWM techniques in comparison to rotatory mobilisation techniques was proven. In order to substantiate these effects further studies with higher sample sizes and follow-ups are necessary.

\section{Einleitung}

Ausgehend von den Daten des Statistischen Bundesamtes wurden im Jahr 2014 in Deutschland ca. 150000 Knietotalendoprothesen-Operationen (Knie-TEP) durchgeführt [21]. Eine Bewältigung des persönlichen Alltags ohne größere Beeinträchtigungen hängt für die betroffenen Patienten unter anderem von der Wiederherstellung einer ausreichenden Beweglichkeit des operierten Gelenks ab. Zur Mobilisation der nach der Operation stark eingeschränkten Beweglichkeit werden physiotherapeutisch unterschiedliche Behandlungs-strategien und Techniken eingesetzt. In der Entzündungsphase stehen dabei abschwellende ebenso wie mobilisierende Maßnahmen für die Knieextension und -flexion im Vordergrund. Mit der abnehmenden Schwellung des direkt nach dem operativen Eingriff zumeist stark ödematösen Knies verbessert sich auch die Gelenkbeweglichkeit.

Mobilisationsziel der postoperativen Krankenhausphase ist meist das Erreichen der vollen Extension und einer Flexion von $90^{\circ}$ [14] [17] [19]. Nicht alle Patienten erreichen in der Akutphase im Krankenhaus diese Mobilisationsziele. Selbst durch das Erreichen der $90^{\circ}$ Flexion sind viele Alltagsaktivitäten (z. B. Ankleiden und Treppenheruntergehen) noch nicht ausreichend zu bewältigen, da hier ein deutlich höherer Flexionsgrad von ca. $110^{\circ}$ nötig ist [8]. Physiotherapeuten müssen daher unter anderem entscheiden, welche Maßnahmen und Techniken im weiteren Rehabilitationsprozess dazu beitragen, die Beweglichkeit optimal und zeitnah zu verbessern, damit Patienten selbstständig ihren Alltag bewältigen können.

Manualtherapeutische Behandlungstechniken gehören zu den klassischen physiotherapeutischen Anwendungen zur Verbesserung der Gelenkbeweglichkeit. Dabei wenden Therapeuten je nach Konzeptausrichtung Gleittechniken in unterschiedlicher Ausführung an. Im manualtherapeutischen Konzept nach Kaltenborn-Evjenth wird die Knieflexion bei einer Anwendung am femorotibialen Gelenkanteil in der Regel durch ein passives Dorsalgleiten auf Grundlage der Konvex-Konkav-Regel (KKR) verbessert [12]. Im Konzept nach Mulligan [1] [2] hängt die Richtung des translatorischen Gleitschubs während der Technik Mobilisation with Movement (MWM) individuell vom Patienten ab. Es wird in die Richtung mobilisiert, die eine schmerzfreie aktive Flexion zulässt [2].

Ob manualtherapeutische Techniken am femorotibialen Gelenk bei Patienten mit implantiertem künstlichem Kniegelenk grundsätzlich zur Verbesserung der Knieflexion beitragen können, ist noch nicht wissenschaftlich belegt. Zwei systematische Literaturrecherchen im Mai 2014 und im August 2015 in den Datenbanken PubMed, Cochrane Library und PEDro ergaben keine Treffer, unabhängig vom manualtherapeutischen Konzept.

Im Extremitätenbereich konnten bisher vor allem positive Effekte der MWM für die Anwendung bei lateraler Epikondylopathie und Distorsionen am Sprunggelenk nachgewiesen werden [3]. Da bisher keine Untersuchungen zur Anwendung der MWM-Technik bei Knie-TEP vorliegen, war das Ziel dieser Arbeit festzustellen, ob Patienten in der ambulanten Rehabilitationsphase von der Anwendung dieser manualtherapeutischen Technik am femorotibialen Gelenkanteil im Vergleich zu einem Standardregime profitieren. Als primärer Nutzen für die Rehabilitanden sollte dabei die Verbesserung der Knieflexion als Therapieziel ausgewertet werden, da sie wie oben aufgeführt - zentraler Bestandteil der Bewältigung des Alltags in der postoperativen Phase von Patienten mit Knie-TEP ist. Der Studie lag daher folgende H1-Hypothese zugrunde: „Die Implementierung der translatorischen Technik MWM innerhalb der physiotherapeutischen Einzelbehandlung bei Patienten mit KnieTEP verbessert die Knieflexion während der ambulanten Rehabilitationsphase wirksamer als die Behandlung nach einem Standardregime ohne Anwendung einer translatorischen Technik.“

\section{Methode}

Durchführungsort der monozentrischen, prospektiven, randomisierten kontrollierten Studie war eine direkt einer orthopädischen Klinik angeschlossene ambulante Rehabilitationseinrichtung. Die Patientenrekrutierung erfolgte im Zeitraum 01.06. bis 03.09.2015. Patienten mit erstmaligem Kniegelenkersatz wurden zufällig auf die Interventions- und Kontrollgruppe verteilt. Die Studie gilt als einfach-verblindet, da nur die Patienten verblindet werden konnten. Diese erhielten mündliche und schriftliche Informationen zum Ziel der Studie, den geplanten Studienablauf und zum Datenschutz.

\section{Ein- und Ausschlusskriterien}

In die Studie eingeschlossen wurden Patienten mit erstmaligem nicht gekoppelten (Unconstrained) Kniegelenkersatz, die zwischen 40 und 85 Jahre alt waren und ausreichende Deutschkenntnisse besaßen. Der Beginn und das Ende der ambulanten Rehabilitationsmaßnahme mussten zwischen dem 8. und 60. postoperativen Tag liegen. Patienten mit einem Prothesenwechsel oder einem gekoppelten Implantat (Semi- und fully constrained) wurden ausgeschlossen ( Tab. 1).

\section{Durchgeführte Interventionen in jeder Gruppe}

Die Studie wurde in das bestehende Therapiekonzept der Rehabilitationseinrichtung integriert, welches den Rehatherapiestandards Hüft- und Knie-TEP der Deutschen Rentenversicherung entspricht [7]. Die Patienten besuchten 3 Wochen lang 5-mal pro Woche die Einrichtung. Nur im Bereich der täglichen physiotherapeutischen Einzelbehandlung kam es für einen Zeitraum von 3-5 Minuten zum Unterschied zwischen Interventions- und Kontrollgruppe.

\section{Interventionsgruppe A:}

Zur Anwendung kamen befundbezogene, individuelle Maßnahmen zur Schmerzlinderung, Resorptionsförderung, Mobilisation, Kräftigung, Koordinationsschulung und Schulung in Aktivitäten des täg- 
Tab. 1 Ein- und Ausschlusskriterien der Studie.

\begin{tabular}{|c|c|}
\hline Einschlusskriterien & Ausschlusskriterien \\
\hline $\begin{array}{l}\text { Patienten mit erstmaligem } \\
\text { Kniegelenkersatz }\end{array}$ & Prothesenwechsel \\
\hline Prothesentyp unconstrained & $\begin{array}{l}\text { Prothesentyp semi- oder fully } \\
\text { constrained }\end{array}$ \\
\hline $\begin{array}{l}\text { 8.- 60. postoperativer Tag } \\
\text { (Interventionszeitraum) }\end{array}$ & $\begin{array}{l}\text { Patienten mit Nervenschädigung an } \\
\text { der unteren Extremität }\end{array}$ \\
\hline Alter $40-85$ Jahre & $\begin{array}{l}\text { vor dem 8. postoperativen Tag oder } \\
\text { nach dem } 60 \text {. postoperativen Tag }\end{array}$ \\
\hline \multirow[t]{7}{*}{$\begin{array}{l}\text { ausreichende Deutsch- } \\
\text { kenntnisse }\end{array}$} & $\begin{array}{l}\text { Patienten mit Thrombose (tiefer } \\
\text { Beinvenenthrombose) oder Embolie }\end{array}$ \\
\hline & $\begin{array}{l}\text { Patienten mit Complex Regional Pain } \\
\text { Syndrome (CPRS) }\end{array}$ \\
\hline & $\begin{array}{l}\text { Patienten mit gleichseitiger Arthritis } \\
\text { des Hüftgelenks/Fußes oder stark } \\
\text { schmerzhaftem Hüftgelenk/Fuß }\end{array}$ \\
\hline & Weichteilossifikationen \\
\hline & ausgeprägte Arthrofibrose \\
\hline & heftige unerklärliche Schmerzen \\
\hline & $\begin{array}{l}\text { Demenzerkrankung oder andere } \\
\text { das Urteilsvermögen erkennbar ein- } \\
\text { schränkende Erkrankungen }\end{array}$ \\
\hline
\end{tabular}

lichen Lebens (ADL). Zur Mobilisation der Knieflexion wurde eine MWM-Technik angewendet. Die Durchführung und Ausgangsstellung richtete sich dabei nach dem Schmerzzustand und der Beanspruchbarkeit des Patienten ( $>$ Abb. 1, > Abb. 2, \Abb. 3). Den Prinzipien für die MWM nach Mulligan [1] [2] folgend, sollten die Therapeuten bei der Durchführung Folgendes beachten [2]:

- Während des akzessorischen Gleitschubs führt der Patient die Bewegung aktiv aus.

- Der Gleitschub wird in die Richtung durchgeführt, in die der Patient die Bewegung schmerzfrei/am schmerzfreiesten ausführen kann.

- Grundsatz für die Intensität des Gleitschubes: So viel, wie zu einer schmerzfreien Verbesserung nötig ist.

- Abhängig von der individuellen Irritierbarkeit des Patienten wird eine Serien- und Wiederholungszahl von zu Beginn 3-mal 10 Wiederholungen, im weiteren Verlauf steigernd auf bis zu 5-mal 10 Wiederholungen angestrebt.

- Abhängig vom Rehabilitationsverlauf werden Ausgangspositionen mit möglichst funktioneller Belastung (z. B. Stand) eingenommen.

\section{Kontrollgruppe B:}

Hier kamen befundbezogene, individuelle Maßnahmen zur Schmerzlinderung, Resorptionsförderung, Mobilisation, Kräftigung, Koordinationsschulung und ADL-Schulung zur Anwendung. Zur Mobilisation der Knieflexion wurden keine MWM-Technik und keine andere translatorische Technik am femorotibialen Gelenkanteil eingesetzt. Translatorische Techniken im Bereich des patellofemoralen Gelenkanteils waren wie in der Interventionsgruppe erlaubt. Zur Mobilisation der allgemeinen Flexionsverbesserung dien-

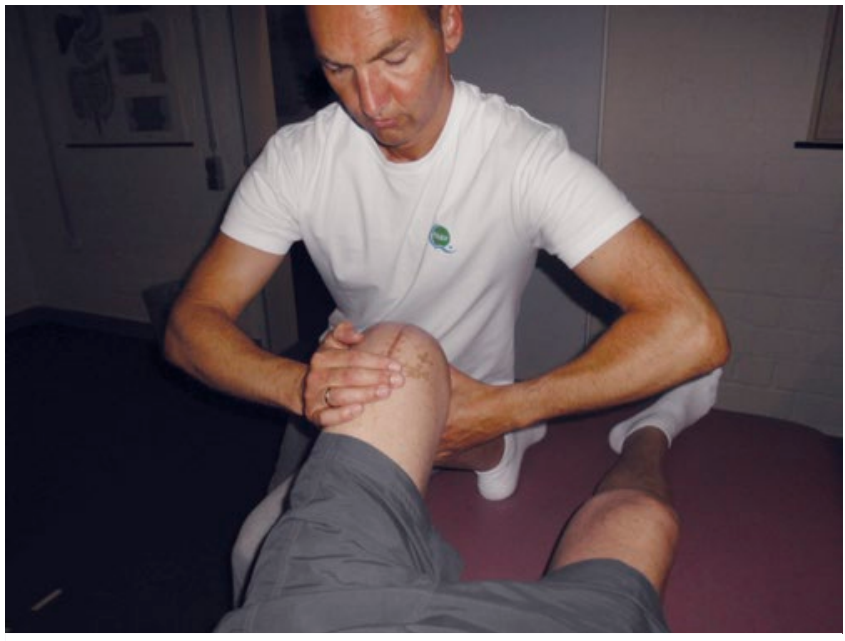

Abb. 1 MWM in Rückenlage. (Quelle: D. Halil)

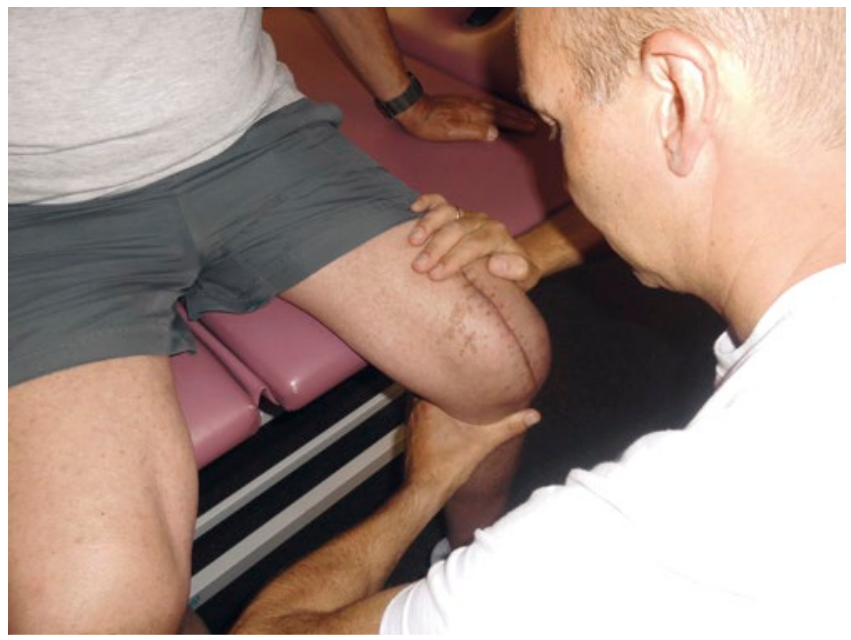

Abb. 2 MWM im Sitzen. (Quelle: D. Halil)

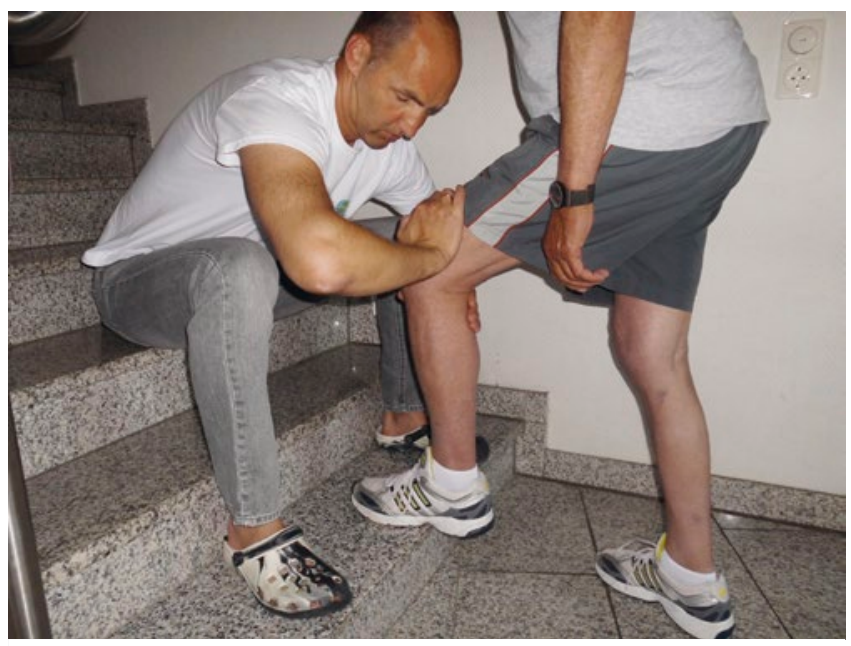

- Abb. 3 MWM im Stand auf der Treppe. (Quelle: D. Halil)

ten rotatorische und/oder funktionelle Übungen, die abhängig von der Behandlungsstrategie des Therapeuten stattfanden. 


\section{Therapeuten}

Alle an der Studie beteiligten Physiotherapeuten waren durch eine abgeschlossene Zusatzausbildung im Bereich der Manuellen Therapie nach dem Kaltenborn-Evjenth-Konzept qualifiziert. Da keiner der Therapeuten über eine manualtherapeutische Ausbildung nach dem Mulligan-Konzept [1] [2] verfügte, wurden sie vor Beginn der Patientenrekrutierung von einem zertifizierten Mulligan-Therapeuten (Certified Mulligan Practitioner, CMP) zweimalig innerbetrieblich instruiert und bekamen zusätzlich eine schriftliche und bebilderte Anleitung zur Durchführung der MWM-Technik ausgehändigt.

\section{Primäre und sekundäre Zielparameter und deren Messmethoden}

Als primärer Zielparameter wurde die Mittelwertdifferenz der aktiven Knieflexion untersucht. Die Messung der Untersucher erfolgte mit dem Standardgoniometer nach einem festgelegten Prozedere. Als sekundäre Zielparameter wurden hinsichtlich der Unterschiede zwischen den Gruppen untersucht: (1) aktive Extension (Standardgoniometer), (2) Funktionsstatus (Knee Injury and Osteoarthritis Outcome Score - KOOS, German version LK1.01) und (3) Schmerz (Numerische Analogskala, NAS).

Die die Therapie durchführenden Therapeuten erhoben auch die aktive Flexion/Extension und die NAS-Werte. Da die Patienten während des Rehabilitationsaufenthaltes vom Beginn bis zum Ende bei einem Physiotherapeuten eingeplant waren, gewährleistete diese meist, dass derselbe Therapeut die Eingangs- und Abschlussmessung vornahm. Das Anmeldepersonal gab den KOOS-Fragebogen aus und sammelte ihn auch wieder ein. Die Daten wurden zu Beginn (T1) und am Ende (T2) der Rehabilitationsmaßnahme erfasst. Der Interventionszeitraum betrug 3 Wochen. Durch einen kurz vor Beginn der Patientenrekrutierung vollzogenen Trägerwechsel der Reha-Einrichtung und den sich dadurch ergebenden personellen und zeitlichen Veränderungen musste abweichend von der Ursprungsplanung auf den Einsatz eines verblindeten Untersuchers und eines Follow-up nach 6 Wochen verzichtet werden.

\section{Randomisierung}

Die Erstellung der Randomisierungslisten erfolgte mithilfe der Internetseite www.randomizer.org. Diese Website erstellt nach Vorgaben des Anwenders Randomisierungslisten zur zufälligen Zuordnung der Patienten auf die Interventions- und Kontrollgruppe. Durch die Vorgabe einer Blockrandomisierung wurden 8 Blöcke à 10 Ziffernreihen generiert. Jeder Block bestand somit aus je 5 zufällig aneinandergereihten geraden und ungeraden Zahlen. Gerade Zahlen wurden der Interventionsgruppe A, ungerade Zahlen der Kontrollgruppe B zugeordnet.

Gemäß den Blockvorgaben wurden Blätter mit der Aufschrift „Gruppe A“ und „Gruppe B“ jeweils einzeln in Umschläge gelegt und verschlossen. Die Umschläge wurden nummeriert und beim Anmeldepersonal der Rehabilitationseinrichtung hinterlegt. Nach Unterzeichnung der Einwilligung zur Studienteilnahme und Kontrolle der Ein- und Ausschlusskriterien durch die Untersucher öffnete das Anmeldepersonal einen Umschlag und stellte die Gruppenzuordnung fest. Unter Beachtung der Geheimhaltung vor den Patienten informierte das Anmeldepersonal die Therapeuten über die Gruppenzuteilung. Nach Abschluss der Patientenrekrutierung ergab sich eine Gruppengröße von jeweils 16 Patienten in der Interventions- und Kontrollgruppe.

\section{Verblindung}

Wie beschrieben, war nur eine einfache Verblindung der Patienten möglich. Da durch kurzfristige personelle Veränderungen in der Rehabilitationseinrichtung kein Untersucher mehr zur Verfügung stand, fungierten die Therapeuten auch als Untersucher. Somit war keine Verblindung der Untersucher gewährleistet. Die Verblindung der Therapeuten ist in manualtherapeutischen Interventionsstudien nicht umsetzbar, da ihnen zu jeder Zeit bewusst ist, welche Technik sie anwenden.

\section{Statistische Methoden}

Die Vorbereitung des Datensatzes und die statistischen Analysen erfolgten mithilfe von Excel 2010, die Berechnungen und Erstellung der Grafiken mit GraphPad Prism6.

Unterschiede in der zentralen Tendenz von Variablen wurden mit parametrischen ( $t$-Test) und nicht parametrischen Verfahren (Mann-Whitney-Test) beim Zwei-Gruppen-Vergleich ermittelt. Bei gepaarten Stichproben kamen die jeweiligen Verfahren für verbundene Stichproben (t-Test, Wilcoxon-Matched-Pairs-Signed-RankTest) zur Anwendung. Zuvor fand mithilfe der Tests auf Normalverteilung nach Shapiro-Wilk sowie D’Agostino und Pearson die Überprüfung statt, ob eine Normalverteilung vorliegt. Das Signifikanzniveau lag bei $5 \%$ (alpha $=0,05)$. Bei fehlenden Daten erfolgte das Vorgehen nach dem Intention-to-treat-Prinzip mit Nutzung der zuletzt vorliegenden Daten.

\section{Ethische Aspekte}

Die Ethikkommission des Deutschen Verbands für Physiotherapie an der Physio-Akademie in Wremen überprüfte das Studienprotokoll auf Einhaltung ethischer Grundsätze und gab es nachfolgend frei.

\section{Ergebnisse}

\section{Ein- und Ausschlüsse von Patienten}

Im Rekrutierungszeitraum wurden der Studie insgesamt 40 Patienten nach endoprothetischer Kniegelenkoperation zugeführt und insgesamt 8 Patienten aus folgenden Gründen ausgeschlossen: fehlende Deutschkenntnisse $(n=3)$, TEP-Wechsel $(n=3)$, fehlende Compliance $(n=1)$ und schlechter Allgemeinzustand $(n=1)$.

Die verbleibenden 32 Patienten wurden nach Studieneinwilligung randomisiert der Interventionsgruppe A (MWM-Gruppe, $n=16$ ) und der Kontrollgruppe B (Standardgruppe, $n=16$ ) zugeteilt. Alle 32 Patienten wurden bezüglich des primären Zielparameters (aktive Flexion) sowie der sekundären Zielparameter aktive Extension und Schmerz zu den Zeitpunkten T1 (Beginn der Rehabilitation) und T2 (Ende der Rehabilitation) erfasst und analysiert. Alle Patienten füllten zum Zeitpunkt T1 den KOOS-Fragebogen aus, 2 Patienten füllten den Fragebogen zum Zeitpunkt 2 nicht aus. Die fehlenden Daten wurden nach dem Intention-to-treat-Prinzip ersetzt. Das bedeutete für die fehlenden T2-Daten des Zielparameters Funktionsstatus, dass je einmal in der Interventions- und in 


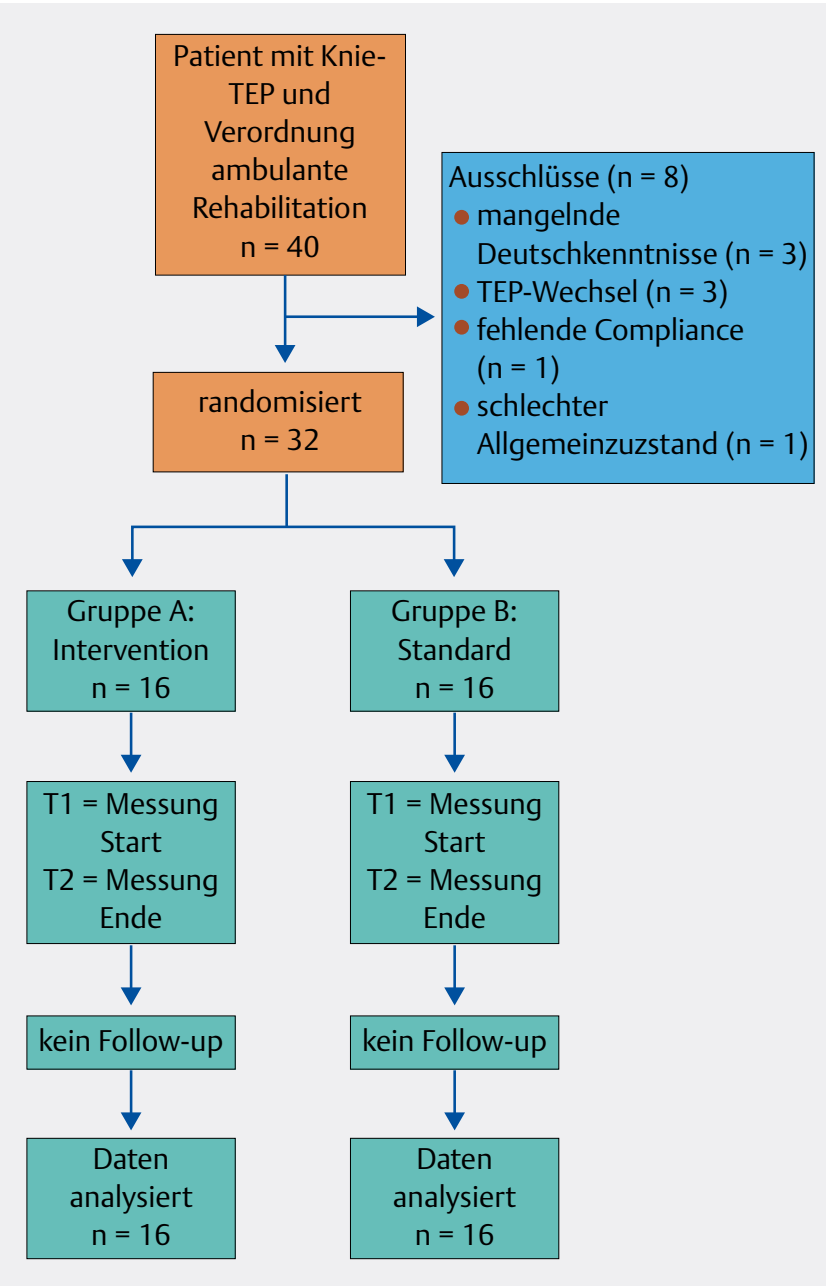

Abb. 4 Patientenfluss während der Studie. (Quelle: D. Halil, graf. Umsetzung: Thieme Gruppe) der Kontrollgruppe die Daten aus dem T1 KOOS-Fragebogen eingesetzt wurden.

\section{Patientenfluss}

Der Patientenfluss während des Studienverlaufs ist in > Abb. 4 detailliert dargestellt.

\section{Charakteristika der Studienteilnehmer zu Studienbeginn}

Die deskriptive Statistik der Variablen Alter, Geschlecht, Gewicht, Größe und BMI für beide Gruppen sowie die primären und sekundären Zielparameter Flexion, Extension, NAS und dem GesamtScore des KOOS-Fragebogens finden sich in $>$ Tab. 2. Alle demografischen Variablen sind gemäß der Testverfahren D'Agostino und Pearson sowie Shapiro-Wilk-Test normalverteilt. Somit konnte der parametrische t-Test als Testverfahren für Unterschiede angewendet werden.

Da in beiden Gruppen zum Zeitpunkt T1 die Variable Extension nach den oben genannten Testverfahren nicht normalverteilt war, kam hier der Mann-Whitney-Test zur Anwendung. Zum Zeitpunkt T1 bestanden keine signifikanten Unterschiede in allen aufgeführten Variablen zwischen den beiden Gruppen.

\section{Primäre Datenanalyse (Hypothesenprüfung)}

Zur Beantwortung der zentralen Fragestellung, ob es bei der Wirksamkeit einen signifikanten Unterschied zwischen den Therapieergebnissen gibt, wurde die Differenz der Werte T2 gegenüber T1 der Goniometermessung für die Flexion ermittelt und anschließend im Gruppenvergleich auf statistische Signifikanz getestet. Im Falle $p<0,05$ gilt die Wirkung einer Therapieform als statistisch signifikant. Noch geringere Werte von $p \leq 0,01$ und $p \leq 0,001$ gelten als statistisch hoch bzw. höchst signifikant [9]. Zur Signifikanzberechnung diente der Mann-Whitney-Test, da die Variable Flexion zum Zeitpunkt T2 in der Kontrollgruppe B nicht normalverteilt war.

- Tab. 2 Messwerte zu Studienbeginn (T1).

\begin{tabular}{|c|c|c|c|}
\hline Variable & Interventionsgruppe $A: n=16$ (SD) & Kontrollgruppe B: $n=16$ (SD) & p-Wert \\
\hline Alter & $71,63(4,57)$ & $70,50(6,57)$ & 0,5783 \\
\hline $\begin{array}{l}\text { Geschlecht: } \\
\text { weiblich (\%) } \\
\text { männlich (\%) }\end{array}$ & $\begin{array}{l}9(56) \\
7(44)\end{array}$ & $\begin{array}{l}8(50) \\
8(50)\end{array}$ & \\
\hline Gewicht (kg) & 83,57 & 92,81 & 0,1905 \\
\hline Größe (cm) & 172,6 & 171,6 & 0,7561 \\
\hline $\mathrm{BMI}$ & 28,1 & 31,49 & 0,1228 \\
\hline primärer Zielparameter: Flexion T1 & $83,13^{\circ}(11,38)$ & $88,75^{\circ}(11,33)$ & 0,1413 \\
\hline $\begin{array}{l}\text { sekundäre Zielparameter: } \\
\text { Extension T1 } \\
\text { NAS T1 } \\
\text { KOOS ges. T1 }\end{array}$ & $\begin{array}{l}-07,19^{\circ}(6,58) \\
5,31(1,82) \\
71,44(18,53)\end{array}$ & $\begin{array}{l}-5,31^{\circ}(5,91) \\
4,38(1,67) \\
67,63(25,12)\end{array}$ & $\begin{array}{l}0,4324 \\
0,1762 \\
0,6288\end{array}$ \\
\hline
\end{tabular}


Flexion $\mathrm{T} 1-\mathrm{T} 2(\mathrm{p}=0,0004)$
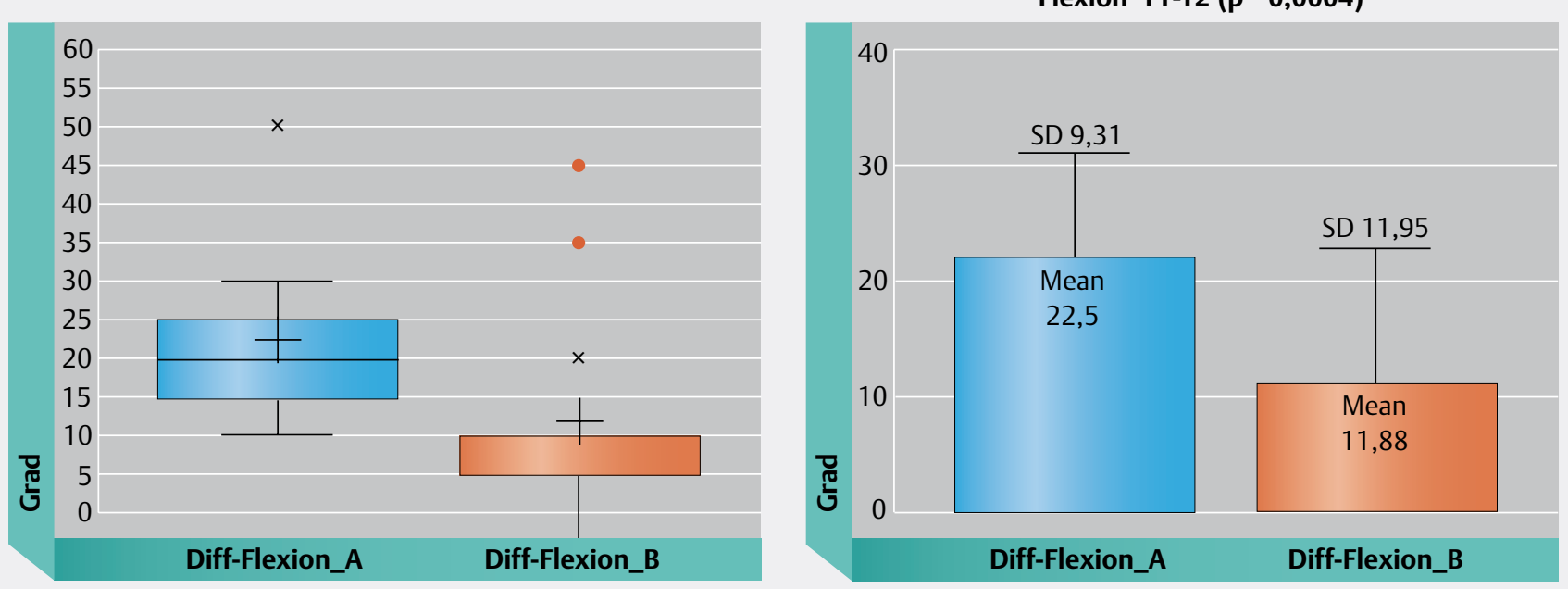

Abb. 5 Differenz des Bewegungsausmaßes T2-T1 in Flexion ( $x=$ Ausreißer; roter Punkt = Extremwert; + = Mean/Mittelwert; SD = Standardabweichung). (Quelle: D. Halil; graf. Umsetzung: Thieme Gruppe)

- Tab. 3 Differenz des Bewegungsausmaßes T2-T1 in Flexion.

\begin{tabular}{|l|l|l|l|l|}
\hline & Mean & SD & $\mathbf{9 5 \% - K I}$ & p-Wert \\
\hline Differenz Flexion Gruppe A T2-T1 & 22,5 & 9,31 & 17,54 & \\
\hline Differenz Flexion Gruppe B T2-T1 & 11,88 & & 27,46 & 0,0004 \\
\hline
\end{tabular}

Bei der statistischen Hypothesenprüfung besteht im Ergebnis ein höchst signifikanter Unterschied zwischen der Interventionsgruppe A (Mean: 22,5; SD: 9,309; 95\%-Konfidenzintervall [KI]: 17,54, 27,46) und der Kontrollgruppe B (Mean: 11,88; SD: 11,95; $95 \%-K I: 5 ; 5,18,25)$. Der P-Wert liegt bei Anwendung des MannWhitney-Test bei $p=0,0004$ ( $\triangleright$ Abb. 5, $\triangleright$ Tab. 3).

In Anbetracht der generierten Hypothesen wird hiermit die $\mathrm{H} 0$-Hypothese abgelehnt und die $\mathrm{H} 1$-Hypothese angenommen.

Innerhalb der Gruppe A kam es zu einer durchschnittlichen Differenz der Flexion von 22,5 (SD: 9,31; $95 \%-K I$ : 17,54; 27,46). In der Gruppe B wurde eine durchschnittliche Differenz der Flexion von 11,88 erreicht (SD: 11,95; $95 \%-K I: 5,5 ; 18,25)$. Gruppe A erzielte somit eine um durchschnittlich $10,62^{\circ}$ höhere Flexionsverbesserung im Zeitraum T1-T2 bei leicht geringerer Streuung der Werte.

\section{Sekundäre Datenanalyse}

\section{Extension}

Da die Variable Extension nach D‘Agostino und Pearson sowie Shapiro-Wilk-Test nicht normalverteilt war, kam zur Prüfung der Unterschiede innerhalb der Gruppen der Wilcoxon-Matched-Pairs-Signed-Rank-Test für gepaarte Daten zu den Zeitpunkten T1 und T2 zur Anwendung. Zur Signifikanzberechnung zu den Unterschieden zwischen den Gruppen pro Zeitpunkt diente der Mann-WhitneyTest als nicht parametrischer Test bei fehlender Normalverteilung.

Innerhalb der Gruppe A kam es zu einer durchschnittlichen Verbesserung der Extension von 6,88 (SD: 6,29; $95 \%-K I: 3,52 ; 10,23$ ).
Gruppe B erzielte eine durchschnittliche Verbesserung der Extension von 5,00 (SD: 6,06; $95 \%$-KI: 1,77; 8,23). Gruppe A erreichte somit eine um durchschnittlich $1,88^{\circ}$ höhere Extensionsverbesserung im Zeitraum T1-T2 bei einer ähnlichen Streuung der Werte. Die Mittelwertdifferenz ist mit $p=0,3609$ nicht signifikant ( Abb. 6, $\triangleright$ Tab. 4).

\section{Schmerz}

Innerhalb der Gruppe A kam es zu einer durchschnittlichen Verbesserung der Schmerzangabe von -3,44 Punkten auf der NAS (SD: 1,79; $95 \%-K I:-4,39 ;-2,49)$. Gruppe B erzielte eine durchschnittliche Verbesserung von -2,13 Punkten auf der NAS (SD: 0,89; $95 \%-K I:-2,60 ;-1,65)$. Gruppe A erreichte somit eine um durchschnittlich 1,31 Punkte höhere Schmerzlinderung im Zeitraum T1T2 auf der NAS. Gruppe B zeigte eine leicht geringere Streuung der Werte. Der Unterschied des Mittelwertes der Gruppe A gegenüber Gruppe B ist mit $p=0,0189$ signifikant ( $>$ Abb. 7, $\triangleright$ Tab. 5).

\section{Funktionsstatus - KOOS-Fragebogen}

Die Auswertung erfolgte für den Gesamt-Score und für folgende Subgruppen-Scores: (1) S = Symptome, (2) P = Schmerz, (3) $A=A D L$ und (4) $Q=$ Beeinflussung der Lebensqualität. Die Subgruppe „Sport und Freizeit“ floss nicht in die Auswertung ein, da die aufgeführten Aktivitäten für Patienten in der untersuchten Zeitspanne nicht relevant sind. Beide Gruppen verbesserten sich signifikant im Gesamt-Score und in allen Subgruppen-Scores. Im Gruppenver- 


\section{Extension T1-T2 $(p=0,3609)$}
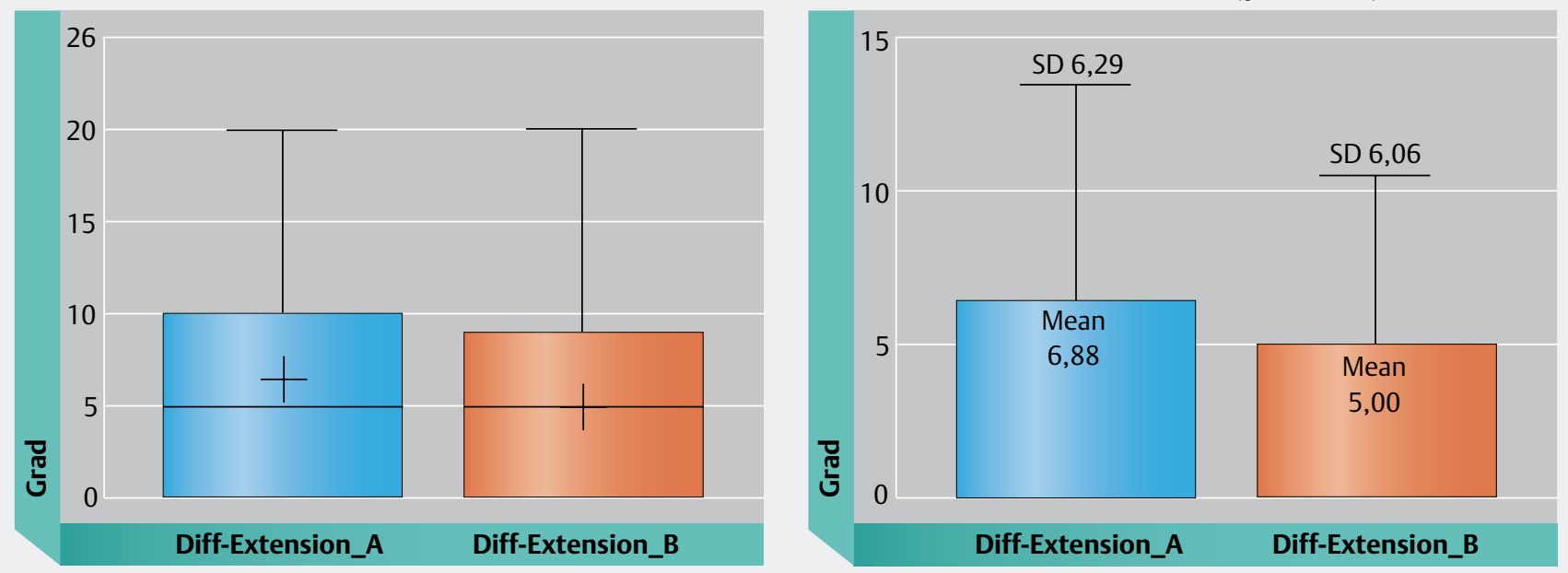

Abb. 6 Differenz des Bewegungsausmaßes T2-T1 in Extension (+ = Mean/Mittelwert; SD = Standardabweichung). (Quelle: D. Halil; graf. Umsetzung: Thieme Gruppe)

- Tab. 4 Differenz des Bewegungsausmaßes T2-T1 in Extension.

\begin{tabular}{|l|l|l|l|l|}
\hline & Mean & SD & $\mathbf{9 5 \% - K I}$ & p-Wert \\
\hline Differenz Extension Gruppe A T2-T1 & 6,88 & 6,29 & 3,52 & \\
\hline Differenz Extension Gruppe B T2-T1 & 5,00 & & 10,23 & 0,3609 \\
\hline
\end{tabular}

gleich ergaben sich weder für den Gesamt-Score noch für die einzelnen Subgruppen-Scores signifikanten Unterschiede.

\section{Diskussion}

Die vorliegende Studie untersuchte die Anwendung der manualtherapeutischen Technik MWM aus dem Mulligan-Konzept [1] [2] in der ambulanten Rehabilitationsphase nach Implantation einer primären Knie-TEP. Obwohl schon Untersuchungen zur Wirkung unterschiedlicher physiotherapeutischer Anwendungen nach KnieTEP vorliegen, war die Applikation und Wirkung manualtherapeutischer Techniken nach Knie-TEP bisher kaum Gegenstand wissenschaftlicher Forschung. Da es noch keine Untersuchung in diesem Setting zur Anwendung der MWM-Technik gibt, hatte dieser monozentrische RCT den Charakter einer Pilotstudie.

\section{Ergebnis der Datenanalyse des primären Zielparameters}

Das Ergebnis der primären Datenanalyse zeigt mit $p=0,0004$ einen statistisch höchst signifikanten Unterschied zwischen der Interventionsgruppe A und der Kontrollgruppe B. Innerhalb der Gruppe A kam es zu einer durchschnittlichen Verbesserung der Flexion von $22,5^{\circ}$, Gruppe B erzielte eine durchschnittliche Verbesserung der Flexion von $11,88^{\circ}$. Gruppe $A$ erreichte somit eine um durchschnittlich $10,62^{\circ}$ höhere Flexionsverbesserung im Zeitraum T1T2 bei leicht geringerer Streuung der Werte.
Bei genauerer Betrachtung der Boxplots in $\mathbf{A}$ Abb. $\mathbf{5}$ zeigt sich, dass $50 \%$ der Patienten in der Interventionsgruppe A eine Flexionsverbesserung zwischen $15^{\circ}$ und $25^{\circ}$ erreichten (gekennzeichnet durch die Box). Die Whisker weisen eine gleichmäßige Verteilung der außerhalb des 25-75\%-Interquartilsabstands liegenden Werte auf. Der bei $50^{\circ}$ auftretende Ausreißer erhöht den Mittelwert der Flexionsverbesserung zwischen T1 und T2 in Gruppe A leicht auf $22,5^{\circ}$. Der Median in der Gruppe A beträgt $20^{\circ}$ (mittlere Linie in der Box).

Die Flexionsverbesserung zwischen T1 und T2 in der Kontrollgruppe B zeigt ein ungleichmäßiges Bild. Hier erreichten $50 \%$ der Patienten eine um $5-10^{\circ}$ verbesserte Knieflexion zwischen T1 und T2. Der fehlende Whisker in höhere Flexionsgrade macht deutlich, dass der Ausreißer (Verbesserung um 20 ${ }^{\circ}$ ) und die beiden Extremwerte $\left(35^{\circ}\right.$ und $\left.45^{\circ}\right)$ für eine Erhöhung des Mittelwerts außerhalb der Box verantwortlich sind. Der Median in der Gruppe B liegt mit $10^{\circ}$ auf der oberen Begrenzung der Box. Ohne die Berücksichtigung der Extremwerte, die nicht mehr als normale Ausreißer gelten [10] hätte sich eine noch deutlichere Differenz zwischen den beiden Mittelwerten zu Gunsten der Gruppe A ergeben.

Warum es zu den Ausreißern und Extremwerten in Gruppe A und B kam, bleibt spekulativ. Auf die Ergebnisse haben sie bei geringen Fallzahlen dennoch großen Einfluss. Möglicherweise spielen hier das Schmerzempfinden oder das Angst-Vermeidungs-Verhalten der betroffenen Patienten zum Zeitpunkt T1 eine Rolle. Ist ein Schmerz sehr groß, oder haben die Patienten große Angst vor 

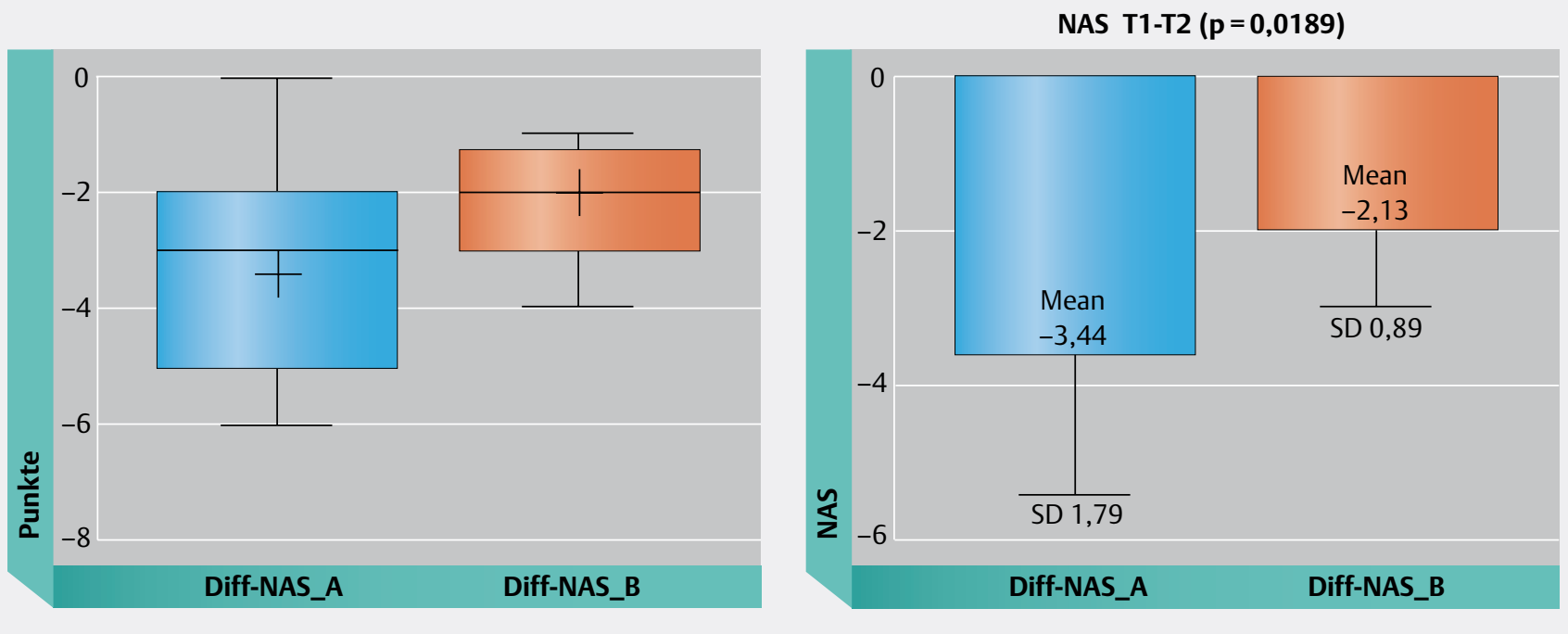

Abb. 7 Differenz des Wertes der numerischen Analogskala T2-T1 (+ = Mean/Mittelwert; SD = Standardabweichung). (Quelle: D. Halil; graf. Umsetzung: Thieme Gruppe)

- Tab. 5 Differenz des Wertes der numerischen Analogskala T2-T1.

\begin{tabular}{|l|l|l|l|l|}
\hline & Mean & SD & 95\%-KI & p-Wert \\
\hline Differenz numerische Analogskala Gruppe A T2-T1 & $-3,44$ & 1,79 & $\begin{array}{l}-4,39 \\
-2,49\end{array}$ \\
\hline Differenz numerische Analogskala Gruppe B T2-T1 & $-2,13$ & & $-2,60$ & 0,0189 \\
\hline
\end{tabular}

einem eventuell bei Flexion auftretenden Schmerz, wird die Goniometermessung vermutlich nicht das momentan maximale Flexionsvermögen des Patienten widerspiegeln. Da einige Patienten nach Knie-TEP eine zeitlich verlängerte Entzündungsphase aufwiesen, könnten hier entsprechend ausgeprägte Entzündungsparameter diese These stützen.

Die Gruppen umfassten jeweils n = 16 Patienten. Für alle statistischen Berechnungen ist festzuhalten, dass die geringe Fallzahl einen großen Einfluss der Ausreißer und Extremwerte auf die statistischen Berechnungen bewirkte. Unter Einbeziehung der Mittelwerte und der Standardabweichung für den primären Zielparameter ergibt die Berechnung der Effektstärke einen Wert von 0,99. Damit wird nach der Empfehlung von Cohen (>0,8 = großer Effekt) trotz der geringen Fallzahl eine große Effektstärke erreicht [16].

Neben der statistischen sollte auch die klinische Signifikanz (Relevanz) der Ergebnisse betrachtet werden. Die klinische Signifikanz beschreibt die Bedeutsamkeit eines Ereignisses oder Befundes für den Alltag von Patienten [16]. Sie beantwortet die Frage, ob von einer Behandlung ein ausreichend großer Behandlungseffekt zu erwarten ist, der in klinischer Hinsicht als „lohnenswert“ zum Nutzen der Patienten gelten kann [9]. Obgleich die klinische Signifikanz für die Knieflexion nach Knie-TEP bisher nicht per Konvention festliegt, wird sie in Studien zur Thematik annähernd übereinstimmend zwischen 5-10 liegend beschrieben [11] [13] [15]. Lenssen et al. [13] sowie Mau-Möller et al. [15] bezeichnen eine Flexions- verbesserung größer $5^{\circ}$, Jakobsen et al. [11] 6,6 $6^{\circ}$ und Rajan et al. [18] $10^{\circ}$ als klinisch signifikant.

Beide Gruppen der vorliegenden Studie erreichten demnach klinisch signifikante Verbesserungen vom Zeitpunkt T1-T2. Beim Vergleich der mittleren Differenz der Flexion von 22,5 in Gruppe A mit $11,88^{\circ}$ in Gruppe B ergibt sich eine Mittelwertdifferenz von $10,62^{\circ}$ zugunsten der Interventionsgruppe A. Diese Differenz kann damit als klinisch signifikante Verbesserung eingestuft werden.

\section{Ergebnisse der Datenanalyse der sekundären Zielparameter}

Bei den sekundären Zielparametern erreichten beide Gruppen signifikante Unterschiede von T1-T2 in allen Variablen. Signifikante Unterschiede zwischen den Gruppen traten nur bei der Schmerzreduktion auf.

Schomacher [20] empfiehlt, die minimale klinisch relevante Reduktion bei geringen Eingangswerten ( $<4$ von 10) bei >0,5 Punkten, bei Eingangswerten $>4$ von 10 bei 2 festzulegen. Beide Gruppen erreichten innerhalb der Gruppen von T1-T2 sowohl eine statistische signifikante wie auch eine klinisch relevante Schmerzreduktion. Die Patienten in Gruppe A erzielten eine im Mittel um 1,31 Punkte größere Schmerzlinderung auf der NAS als Gruppe B. Dieser Unterschied ist mit $\mathrm{p}=0,0189$ statistisch signifikant.

Ob diese Mittelwertdifferenz nach der Empfehlung von Schomacher [20] klinisch relevant wäre, hängt vom T1-Wert der jeweiligen Patienten ab. In Gruppe A hatten 3 und in Gruppe B 5 Patien- 
ten zum Zeitpunkt T1 einen NAS-Wert von $\leq 3$. Für diese Patienten wäre eine Veränderung von 1,31 Punkten nach der Empfehlung von Schomacher [20] relevant, da sie mehr als 0,5 Punkte beträgt.

Mulligans „Prinzip der sich aus der schmerzfreien Bewegungsrichtung ableitenden Behandlungsrichtung und der schmerzfreien Ausführung der Techniken“ sieht vor, dass zu keinem Zeitpunkt der Technikanwendung ein Schmerz auftreten darf [1] [2]. Die passive Zusatzbewegung erfolgt immer in die Richtung, in die der Patient während der vom Therapeuten durchgeführten Bewegung Schmerzfreiheit signalisiert. Die rotatorische Mobilisationstechnik in Gruppe B lag im Ermessen der Therapeuten und wurde nicht dokumentiert. Demnach ist keine Aussage zur Schmerzfreiheit oder Schmerzhaftigkeit der applizierten Techniken in der Kontrollgruppe möglich. Eventuell trug Mulligans [1] [2] Prinzip dazu bei, dass eine effektivere Schmerzlinderung in der Interventionsgruppe erreicht wurde. Weitere Untersuchungen müssen zeigen, ob MWM als probate Technik zur Schmerzlinderung bei Patienten nach KnieTEP infrage kommt.

Der KOOS hat nach Knieverletzungen eine hohe Test-Retest-Reliabilität mit Intraclass-Korrelationskoeffizient-Werten (ICC) für die verwendeten Subskalen von 0,75- 0,97 [4]. Eine Studie zur holländischen Übersetzung untersuchte in einer Gruppe auch Patienten nach Knie-TEP-Revision und stellte für alle Subskalen ICC >0,86 mit Ausnahme in der Subskala Sport (ICC: 0,45$)$ fest [5]. Ungeachtet des deutlichen Unterschieds im Flexionsvermögen des operierten Knies zwischen den Gruppen der vorliegenden Studie wurde die subjektive Verbesserung des Funktionsstatus des Knies von Patienten beider Gruppen ähnlich gut bewertet. Dies wirft die Frage auf, ob die Responsivität (Empfindlichkeit) der deutschen KOOS-Version für eine realistische Bewertung ausreichend hoch ist.

Der Unterschied des BMI zwischen den Gruppen ist statistisch nicht signifikant. Nach der Einteilung der Deutschen Adipositas Gesellschaft gelten die Patienten der Gruppe A mit einem durchschnittlichen BMI von 28,1 als übergewichtig, die Patienten der Gruppe B mit 31,49 als adipös Grad 1 [6]. Möglicherweise stellte ein hoher BMI einen limitierenden Faktor für die aktive Flexion in der Rehabilitationsphase dar und trug zu einem schlechteren Flexionsergebnis der Kontrollgruppe bei.

\section{Limitationen}

\section{Mobilisationstechnik in der Kontrollgruppe B}

In der Kontrollgruppe kamen zur Mobilisation keine translatorischen Techniken zum Einsatz. Die durchgeführten Anwendungen waren abhängig von der Behandlungsstrategie des jeweiligen Therapeuten. Somit erfolgte ein Vergleich der MWM-Technik in der Interventionsgruppe mit einem inhomogenen Mobilisationskonzept in der Kontrollgruppe. Demnach sind nur Rückschlüsse bezüglich eines Vergleichs zwischen der translatorischen MWM-Technik und einer unspezifischen rotatorischen Mobilisationsstrategie in der Kontrollgruppe zulässig.

\section{Verblindung und Follow-up}

Die Studienplanung sah zunächst eine Verblindung der Untersucher vor. Der kurzfristig anberaumte Trägerwechsel der Rehabilitationseinrichtung mit in der Folge ausgelöstem Personalwechsel/-abbau und zeitlichen Verzögerungen ließ die Umsetzung dieser wichtigen Maßnahme zur Steigerung der internen Validität nicht zu. Da die behandelnden Therapeuten die Messungen des Bewegungsausmaßes und des Schmerzes durchführten, ist eine mögliche Beeinflussung der Messergebnisse seitens der Therapeuten nicht auszuschließen. Da die Studie im Rahmen einer Master-Arbeit entstand, musste aus zeitlichen Gründen auf das geplante Follow-up nach 6 Wochen verzichtet werden. Somit ist keine Aussage zu mittel- oder längerfristigen Effekten der Interventionen möglich.

\section{Stichprobengröße}

Ausgehend von der Fallzahlplanung wurde eine Stichprobengröße von $n=26$ pro Gruppe angestrebt. Bedingt durch die oben aufgeführten Umstände und die damit verkürzte Patientenrekrutierungszeit ließ sich die angestrebte Fallzahl nicht erreichen. Es ist nicht auszuschließen, dass sich die Ergebnisse bei einer höheren Fallzahl abweichend oder differenzierter dargestellt hätten.

\section{Schmerzmitteleinahme}

Die Studienplanung sah nicht vor, die individuelle Schmerzmitteleinnahme der Patienten zu erfassen. Es kann nicht ausgeschlossen werden, dass diese Einfluss auf sämtliche primäre und sekundäre Zielparameter hatte.

\section{Schlussfolgerungen}

Aus Sicht der klinischen Signifikanz ist die Implementierung der translatorischen MWM-Technik in die physiotherapeutische Einzelbehandlung zur Verbesserung der Flexion nach primärer KnieTEP als sinnvoller und lohnenswerter Behandlungsansatz anzusehen. Die höchst signifikanten Flexionsverbesserungen in der Interventionsgruppe zeigen unter Berücksichtigung der genannten Limitierungen eine Überlegenheit von MWM gegenüber unspezifischen rotatorischen Techniken. Auf welcher Ursache sie beruhen, ist an dieser Stelle nicht ausreichend zu beantworten. Mulligans [1] [2] Prinzipien zur Durchführung der MWM müssten in diesem Zusammenhang Gegenstand weiterer Untersuchungen sein. Aus manualtherapeutischer Sicht ist vor allem die Effektivität des translatorischen Gleitschubs interessant. Möglicherweise spielen hier die auf Mulligans [1] [2] Prinzipien basierenden Aspekte der schmerzfreien Mobilisation und die Wahl einer möglichst funktionellen Ausgangsstellung eine entscheidendere Rolle.

Aus den vorliegenden Ergebnissen lässt sich die Empfehlung ableiten, die MWM-Technik in der rehabilitativen Phase nach KnieTEP in die physiotherapeutische Einzelbehandlung zu integrieren. Zur evidenzbasierten Absicherung dieser Empfehlung sollten weitere Studien folgende Aspekte und Fragestellungen untersuchen: (1) Höhere Fallzahlen mit Verblindung der Untersucher und Berücksichtigung der Schmerzmitteleinnahme; (2) Untersuchung der mittel- und langfristigen Effekte der MWM durch angemessene Follow-up-Untersuchungen; (3) Zeigt sich bei Anwendung der MWM-Technik zur Mobilisation des Knies nach primärer Knie-TEP eine Translationsrichtung (medial, lateral, dorsal, ventral), von der die Patienten bevorzugt profitieren? und (4) Ist der manuelle translatorische Gleitschub der entscheidende Aspekt zur Erlangung positiver signifikanter Ergebnisse bei der Anwendung der MWM oder ist er nur in Kombination mit Mulligans [1] [2] weiteren Prinzipien effektiv? 
[1] Bessler ], Beyerlein C. Manuelle Therapie nach Mulligan. Stuttgart: Thieme; 2015

[2] Beyerlein C. Therapiekonzepte in der Physiotherapie - Mulligan. Stuttgart: Thieme; 2009

[3] Bisset L, Hing W, Vicenzino B. A systematic review of the efficacy of MWM (Chapter 3). In: Vicenzino B, Hing W, Rivett D, Hall T. Mobilisation with Movement - The art and the science. Sydney: Elsevier; 2011

[4] Collins NJ, Misra D, Felson DT, Crossley KM, Roos EM. Measures of knee function: International Knee Documentation Committee (IKDC) Subjective Knee Evaluation Form, Knee Injury and Osteoarthritis Outcome Score (KOOS), Knee Injury and Osteoarthritis Outcome Score Physical Function Short Form (KOOS-PS), Knee Outcome Survey Activities of Daily Living Scale (KOS-ADL), Lysholm Knee Scoring Scale, Oxford Knee Score (OKS), Western Ontario and McMaster Universities Osteoarthritis Index (WOMAC), Activity Rating Scale (ARS), and Tegner Activity Score (TAS). Arthritis Care Res 2011; 63 (Suppl11): S208-S228

[5] De Groot IB, Favejee M, Reijman M, Verhaar J, Terwee CB. The Dutch version of the knee injury and osteoarthritis outcome score: A validation study. Health Qual Life Outc 2008; 6: 16

[6] Deutsche Adipositas Gesellschaft. Was ist der Body Mass Index. www. adipositas-gesellschaft.de/mybmi/index.php (30.08.2015)

[7] Deutsche Rentenversicherung Bund. Reha-Therapiestandards Hüftund Knie-TEP. Stand Januar 2011. www.deutsche-rentenversicherung. de/Allgemein/de/Navigation/3_Infos_fuer_Experten/01_Sozialmedizin_Forschung/02_reha_qualitaetssicherung/reha_therapiestandards/ indikationen/rts_hueft_knie_tep_node.html (30.08.2015)

[8] Figgie HE, Goldberg VM, Heiple KG, Moller HS, Gordon NH. The influence of tibial-patellofemoral location on function of the knee in patients with the posterior stabilized condylar knee prosthesis. J Bone Joint Surg Am 1986; 7: 1035-1040

[9] Herbert RD. Beurteilung der klinischen Signifikanz von Studienergebnissen. In: Scherfer E, Bossmann T. Forschung verstehen. Ein Grundkurs in evidenzbasierter Praxis. München: Pflaum; 2011
[10] Holling H, Gediga G. Statistik - Deskriptive Verfahren. Göttingen: Hogrefe; 2011

[11] Jakobsen TL, Christensen M, Christensen SS, Olsen M, BandholmT. Reliability of knee joint range of motion and circumference measurements after total knee arthroplasty: does tester experience matter? Physiother Res Int 2010; 15: 126-134

[12] Kaltenborn F, Evjenth O, Baldauf Kaltenborn T. Manuelle Therapie nach Kaltenborn. Untersuchung und Behandlung. Oslo: Norlis; 1999

[13] Lenssen AF, Crijns YHF, Waltje EMH et al. Effectiveness of prolonged use of continuous passive motion (CPM) as an adjunct to physiotherapy following total knee arthroplasty: design of a randomised controlled trial. BMC Musculoskelet Disord 2006; 7: 15

[14] List M (Hrsg). Physiotherapie in der Traumatologie. Heidelberg: Springer; 2009

[15] Mau-Moeller A, Behrens M, Finze S et al. The effect of continuous passive motion and sling exercise training on clinical and functional outcomes following total knee arthroplasty: a randomized active-controlled clinical study. Health Qual Life Outcomes 2014; 9: 12-68

[16] Mehrholz ], Supp G. Wissenschaft transparent. Klinische Studien verstehen. Rosshaupten; McKenzie Institut; 2013

[17] Mockford B], Thompson NW, Humphreys P, Beverland DE. Does a standard outpatient physiotherapy regime improve the range of motion after primary total knee arthroplasty? J Arthroplasty 2008; 23: $1110-1114$

[18] Rajan RA, Pack Y, Jackson H, Gillies C, Asirvatham R. No need for outpatient physio-therapy following total knee arthroplasty: a randomized trial of 120 patients. Acta Orthop Scand 2004; 75: 71-73

[19] Schabus R, Bosina E (Hrsg). Das Knie - Diagnostik, der Ratgeber für das verletzte Knie. Therapie und Rehabilitation bei Verletzungen des Kniegelenks. Wien: Springer; 2007

[20] Schomacher]. Gütekriterien der visuellen Analogskala zur Schmerzbewertung. physioscience 2008; 4: 125-133

[21] Statistisches Bundesamt. www.destatis.de/DE/ZahlenFakten/GesellschaftStaat/Bevoelkerung/Bevoelkerung.html (31.05.2016) 